\title{
Sensitivity to change of the UK Diabetes and Diet Questionnaire in a specialist weight management service
}

\section{Abstract}

The UK Diabetes and Diet Questionnaire (UKDDQ) is a brief dietary questionnaire developed for people with, or at high risk of, type 2 diabetes $^{(1)}$. It consists of 20 items scored from $0-5$ ( 0 healthiest, 5 least healthy). It has been demonstrated to be reliable and compares well with food diaries. A study evaluating sensitivity to change of the UKDDQ was undertaken at the specialist weight management service (WMS) at Musgrove Park Hospital, Taunton. WMS patients receive an initial appointment with an endocrinologist and dietitian, followed by referral to group sessions or dietary advice. Follow up appointments with a WMS specialist occur over the next 26 weeks. Adults attending the service were recruited for the study between September 2016-March 2017. Participants completed the UKDDQ in the waiting room at the initial appointment and the first follow-up. Diabetes status, binge eating assessment and weight at both timepoints were obtained from WMS records. UKDDQ scores from $0-5$ were calculated for each participant by summing the score for each item and dividing by 20. Change in UKDDQ scores and absolute and percentage change in weight between appointments were calculated. Paired sample t-tests were used to test differences in means for UKDDQ scores and weight from baseline to follow up. Multivariable regression analysis was used to examine associations between changes in the UKDDQ scores and percentage change in weight. The model was adjusted for age, gender, follow up time, diabetes status and binge eating. Forty-eight White British participants completed the study (67\% women, 37\% type 2 diabetes, $29 \%$ binge eating). Baseline mean weight was $132.4(29.4) \mathrm{kg}$, mean BMI $46.5(7.7) \mathrm{kg} / \mathrm{m}^{2}$ and mean UKDDQ score was $1.39(0.49)$. Participants lost weight $(-2.4$ $(6.9) \mathrm{kg}, \mathrm{p}=0.006)$ and the UKDDQ score improved between baseline and follow up $(-0.27(0.53), \mathrm{p}=0.001)$. The mean percentage weight change was $-1.6(5.0) \%$. There was some evidence that a one point deterioration in healthy eating was associated with a $2.4(-0.2$ to 5.1$) \%$ increase in weight $(\mathrm{p}=0.072)$. The UKDDQ can measure dietary change in people attending a specialist WMS and there is some evidence that a change in score is associated with a change in weight. The UKDDQ could be used as an outcome measure in these services.

\section{Conflict of Interest}

There is no conflict of interest

\section{References}

1. England, CY et al.. (2016) Development of a brief, reliable and valid diet assessment tool for impaired glucose tolerance and diabetes: the UK Diabetes and Diet Questionnaire. Public Health Nutrition, 1-9. 